\title{
NECESSIDADES NO CUIDADO HOSPITALAR DO LESADO MEDULAR
}

\author{
NEEDS REGARDING THE HOSPITAL CARE PROVIDED TO SPINAL CORD INJURED PATIENTS
}

Nely R Sartori ${ }^{1} \&$ Márcia RAC Melo²

\begin{abstract}
'Bolsista de Iniciação Científica do Pibic/CNPq/USP. ${ }^{2}$ Docente do Departamento de Enfermagem Geral e Especializada. Escola de Enfermagem de Ribeirão Preto - USP

Correspondéncia: Márcia Regina A. Costa Meio - Avenida Bandeirantes, 3900 14040-902 — Ribeirão Preto — SP, mracmelo@eerp.usp.br
\end{abstract}

SARTORI NR \& MELO MRAC. Necessidades no cuidado hospitalar do lesado medular. Medicina, Ribeirão Preto, 35: 151-159, abr./jun. 2002.

Resumo: Modelo de estudo: Estudo retrospectivo e documental. Objetivo: Identificar a incidência das complicações e quais as ações assistenciais a serem desenvolvidas pela equipe de enfermagem ao paciente com lesão medular. Método: Foram analisados os prontuários dos pacientes com lesão medular, segundo a classificação do CID 10, no período compreendido entre 1997 a 2000; os dados foram obtidos através do Serviço de Arquivo Médico (SAME) da instituição, a partir da solicitação de um relatório de morbidade; elaborou-se um instrumento que continha a identificação do paciente, dados básicos da história clínica e as alterações fisiológicas apresentadas, bem como os dias após a internação em que elas ocorreram e quais os cuidados prestados pela equipe de enfermagem; os dados foram agrupados e analisados através de tabela e quadro. Resultados: Observou-se que os pacientes com lesão medular ficam realmente susceptíveis às complicações, como infecções, bexiga neurogênica, íleo paralítico, priapismo, trombose venosa profunda, entre outras; também foi constatada, através das anotações da equipe de enfermagem, a inexistência de cuidados específicos a tais complicações, ocorrendo apenas os cuidados básicos e as ações de natureza propedêutica e terapêutica complementares ao ato médico. Neste estudo, também se observou que as anotações das orientações da alta hospitalar, não apareceram em seis prontuários, e que, em nove, as anotações não eram especificas aos cuidados que o paciente e seus cuidadores necessitariam em seu domicilio a fim de prevenir novas intercorrências. Considerações: A realização deste estudo possibilitou-nos uma visão mais ampla do contexto que envolve o cuidado ao paciente com lesão medular. Acredita-se ser importante um programa de educação continuada em enfermagem, de modo a garantir a atualização do conhecimento para toda a equipe, das necessidades de cada paciente e etapa pela qual está passando.

Unitermos: Traumatismo de Medula Espinhal. Assistência de Enfermagem. Enfermagem Ortopédica.

\section{INTRODUÇÃO}

Reconhecendo como de fundamental importância o conhecimento das mudanças do perfil de morbidade hospitalar para o acompanhamento de estratégi- as adequadas de organização do trabalho e atualização do conhecimento para embasar o cuidado prestado, Melo et al. (2000) ${ }^{1}$ desenvolveram um estudo em unidade de internação de Ortopedia e constataram um número considerável de altas hospitalares a portadores de lesão medular pós trauma. 
Com fundamento em tais dados, busca-se, com este estudo, gerar conhecimento sobre o cuidado hospitalar do paciente com lesão medular, que sustente a prevenção, promoção, recuperação e manutenção da saúde dos portadores da referida afecção.

Considerando-se tais aspectos, faz-se necessário (re)estruturar e implementar alternativas com vistas à qualificação da assistência de enfermagem prestada, o que justifica a realização deste estudo, que é parte do projeto de pesquisa. "Reconstruindo a prática assistencial: buscando caminhos para a administração em enfermagem".

O paciente com lesão medular é aquele que sofreu uma lesão traumática na medula espinhal devido a um abalo, compressão ou ruptura total ou parcial da transmissão medular, causando, assim, uma alteração da função normal da medula ${ }^{2}$. A medula espinhal é parte do Sistema Nervoso Central, contida na coluna vertebral, e "qualquer dano causado por agente fisico (ferimento por arma de fogo) ou choque violento (quedas ou acidentes automobilísticos) pode causar a perda permanente da sensibilidade e/ou motricidade, levando o indivíduo a uma tetraplegia ou paraplegia"3.

A medula espinhal é formada por segmentos e raízes nervosas, e essas raízes emergem da medula no nível de cada segmento. "São oito cervicais, doze torácicos, cinco lombares e cinco sacrais. Os segmentos de C5 a TI inervam os membros superiores, enquanto os segmentos de T12 a S4 se destinam aos membros interiores" 4 .

Durante a fase aguda da lesão medular, que compreende o período imediato após o trauma, após o choque medular e após a adaptação do paciente às lesões dos tratos espinhais, exige-se da equipe médica e de enfermagem da instituição uma atenção especial e um tratamento intenso e até, muitas vezes, cirúrgico. A fase pode durar de três a quatro semanas, estendendo-se até três meses 5 .

O lesado medular apresenta uma das mais graves incapacidades que pode acometer um indivíduo, pois torna-o um paciente com alterações motoras e sensitivas, além de ter desregulada suas funções fisiológicas, passando a apresentar alterações respiratórias, vasculares, da atividade sexual, urinárias e intestinais. Dependendo da altura da lesão, este indivíduo pode apresentar um quadro clínico mais ameno ou mais grave, pois quanto mais alta a lesão, maior é a possibilidade de produzir alterações graves e complicações imprevisíveis, principalmente durante a fase aguda da lesão.
Como esses pacientes apresentam várias alterações de suas funções fisiológicas, tornam-se deste modo mais suceptíveis a infecções e sépsis, e é esta a maior causa de morbi-mortalidade nestes pacientes. Esses pacientes necessitam de uma avaliação completa e tratamento imediato, pois, qualquer tipo de infecção pode ser ameaçadora à vida

As principais alterações fisiológicas que ocorrem com esse paciente ${ }^{5 / 7}$ são:

\section{- Alterações respiratórias}

$\mathrm{Na}$ lesão da coluna alta, a causa principal de morte é a insuficiência respiratória, pois os músculos que contribuem para a respiração que são os abdominais e os intercostais são inervados pelos segmentos que vão de T1 a T11, e o diafragma que é inervado pelo nervo frênico que fica na altura do segmento C35. Estes pacientes apresentam diminuição da capacidade vital, fluxo respiratório forçado, aumento do trabalho respiratório e acúmulo de secreções.

\section{- Alterações térmicas}

O desregulamento térmico acontece devido ao bloqueio nervoso aferente ao centro térmico e nervoso eferente responsável pelas respostas de defesa como a piloereção, vasoconstrição e tremores. Podem apresentar tanto hipertermia como hipotermia.

\section{- Trombose venosa profunda ou tromboilebite}

É um distúrbio comum das veias, onde se formam trombos. Esses trombos podem se desprender e causar uma embolia pulmonar, levando o paciente ao óbito. Esta patologia costuma aparecer sessenta dias após a instalação da lesão medular.

\section{- Hipotensão ortostática ou postural}

A pressão arterial cai após o paciente ficar em pé ou sentado, sendo acompanhada de zonzeira, tontura ou síncope. A frequiência cardíaca não aumenta o suficiente para compensar os efeitos da gravidade durante a posição ortostática.

\section{- Disreflexia autonômica}

É uma condição clínica freqüentemente associada a um alto índice de morbi-mortalidade, também é conhecida como Síndrome de Hiperatividade do Sistema Nervoso Autônomo Simpático e Parassimpático. E desencadeada por estímulos cutáneos (escaras, dobras nas roupas, excesso de calor ou frio, mudanças de posição, entre outros) ou estímulos viscerais da 
bexiga e intestino (retenção urinária levando à distensão vesical, obstrução ou pinçamento do catéter vesical de demora, litíases, infecções no trato urinário, lesões no pênis e bolsa escrotal, distensão do cólon ou do reto por constipação fecal, acúmulo de gazes, enemas ou toques retais). Os sinais e sintomas mais freqüentes durante essa crise é afasia, perda de consciência e coma, convulsões, dispnéia, ansiedade, náuseas, priapismo e bradicardia.

\section{- Alterações gastrointestinais e metabólicas}

$\mathrm{O}$ paciente lesado medular tem uma tendência a desenvolver uma distensão visceral devido a uma redução do peristaltismo intestinal e a uma hipotonia gástrica, e isso pode ser um sério risco para o desenvolvimento de úlceras gástricas e duodenais com sangramento, uma vez que há uma hiperatividade simpática, que produzirá um aumento da secreção gástrica.

\section{- Alterações vesicais}

O efeito da lesão na coluna sobre a bexiga vai depender da altura da lesão, do grau de dano à medula e extensão de tempo de lesão. O lesado medular pode apresentar tanto uma bexiga reflexa ou não reflexa, mas em qualquer um desses casos, o indivíduo perde o controle da micção.

\section{Úlceras de pressão}

É muito comum o paciente lesado medular apresentar úlcera de pressão, principalmente na região sacral e/ou grande trocânter. Costuma se desenvolver nas regiões de proeminência óssea, que estão constantemente expostas a pressão não aliviada. Os fatores que contribuem para o surgimento das úlceras são: perda sensorial permanente sobre as áreas de pressão, imobilidade, perda da função protetora da pele, umidade e/ou calor excessivo, irritação da pele, más condições de higiene, incontinência fecal e urinária e outras.

Nosso interesse em realizar este trabalho se deu frente a dificuldades no cuidado com esses pacientes e em contribuir com novos conhecimentos nessa área ou reiterar os já existentes. Este estudo tem como objetivos:

- Identificar a frequiência das complicações que acometem o lesado medular.

- Identilicar quais são as ações assistenciais desenvolvidas pela equipe de enfermagem durante a internação do lesado medular.

\section{MÉTODO}

O estudo foi realizado no Hospital das Clínicas de Ribeirão Preto - HCRP, nível terciário, com estrutura adequada (recursos humanos, materiais e tecnológicos) para os pacientes que exigem um alto nível de complexidade.

Tratou-se de um estudo documental que é aquele realizado a partir de documentos sob a forma de textos, imagens, sons, sinais em papel/madeira/pedra e outros. Ainda são considerados os documentos oficiais, como editoriais, leis, atas, e os documentos jurídicos, oriundos de cartórios, inventários, escrituras de compra e venda isto é, cientificamente autênticos (não fraudados).

Os dados foram coletados através de consulta a prontuários de pacientes que se encontravam na sala de Serviço de Arquivos Médicos (SAM) e da sala de Arquivos Semi Ativos (ASA) do IICRP. Estes prontuários foram obtidos após solicitação ao funcionário responsável pela sala de Levantamentos e Prontuários Médicos através do preenchimento de um formulário informando o Código Internacional das Doenças $(\mathrm{CID})^{9}$ e o período desejado.

A coleta de dados iniciou-se em janeiro de 2001 e foi concluída em julho do mesmo ano.

Através do levantamento solicitado, foram obtidos no Relatório de Morbidade por patologia de 19972000 , um total de 123 prontuários. Deste total, 80 referiam-se a afecção solicitada, e destes 17 indicavam óbito, um prontuário não foi encontrado e apenas 15 deles se reportavam realmente à afecção pesquisada, que é a de traumatismo de coluna vertebral com lesão medular.

O instrumento de coleta de dados constou de três partes, a primeira se reporta aos dados pessoais e o diagnóstico principal dos pacientes. A segunda compõe-se de dados sobre a história clínica e a última parte sobre as intercorrências desenvolvidas e as ações de enfermagem realizadas por essa equipe, as quais foram preenchidas conforme os dados encontrados nos prontuários dos pacientes.

\section{APRESENTAÇÃO DOS RESULTADOS}

Após a coleta e várias leituras dos dados, eles foram agrupados de acordo com a sequiência do instrumento, ou seja, o diagnóstico principal do paciente, os dados da história clínica e as intercorrências desenvolvidas, conforme as Tabelas I, II, III, IV, V, VI e Quadro 1, .a seguir apresentados. 


\begin{tabular}{|c|c|c|c|c|c|c|}
\hline \multirow[t]{2}{*}{ Idade } & \multicolumn{2}{|c|}{ Masculino } & \multicolumn{2}{|c|}{ Ferririno } & \multicolumn{2}{|c|}{ Total } \\
\hline & $\mathrm{N}^{\circ}$ & $\%$ & $\mathrm{~N}^{\circ}$ & $\%$ & $\mathrm{~N}^{\circ}$ & $\%$ \\
\hline $\begin{array}{l}16-30 \text { anos } \\
31-45 \text { anos }\end{array}$ & $\begin{array}{r}11 \\
4\end{array}$ & $\begin{array}{l}73,4 \\
26,6\end{array}$ & - & - & $\begin{array}{r}11 \\
4\end{array}$ & $\begin{array}{l}83,4 \\
26,6\end{array}$ \\
\hline TOTAL & 15 & 100,0 & - & - & 15 & 100,0 \\
\hline
\end{tabular}

\begin{tabular}{|l|r|r|}
\hline \multicolumn{2}{|l|}{ Tabela II - Dis trh uição numé ric a e percentual dos } \\
pacie ntes do HCRP, com traumatis mo raquimedular, \\
segundo estado ciril, no periodo de 1997-2000 - Rheirão \\
Preto, 200l.
\end{tabular}

\begin{tabular}{|c|c|c|}
\hline \multicolumn{3}{|c|}{$\begin{array}{l}\text { Tabeh III - Distrbuçãa dos pacientes do HCRP, segundo } \\
\text { unidade de internação, no perío do conpre-endido entre } \\
\text { 1997-2000 - Rheirão Preto, } 2001 \text {. }\end{array}$} \\
\hline CLÍNICA & $\mathrm{N}^{\circ}$ & $\%$ \\
\hline $\begin{array}{l}\text { Ne urobgia } \\
\text { Ortopedia } \\
\text { Outras }\end{array}$ & $\begin{array}{l}5 \\
9 \\
1\end{array}$ & $\begin{array}{r}33,3 \\
60,0 \\
6,7\end{array}$ \\
\hline TOTAL & 15 & 100,0 \\
\hline Forte: pror & & \\
\hline
\end{tabular}

Tabeh IV - Dis trburção dos pacientes do HCRP, segundo nírel da lesão, no peńodo compreendido entre 1997-2000 -Rظeirão eo 2001.

\begin{tabular}{|l|r|r|}
\hline NÍVEL DA LESÃO & $N^{\circ}$ & $\%$ \\
\hline C6-C7 & 2 & 13,3 \\
T4-T5 & 2 & 13,3 \\
T5-T6 & 1 & 6,7 \\
T8-T12 & 1 & 6,7 \\
T10 & 1 & 6,7 \\
T11-T12 & 6 & 39,9 \\
L1-L4 & 1 & 6,7 \\
Não especificado & 1 & 6,7 \\
\hline TOTAL & 15 & 100,0 \\
\hline Forte: prontuários de paciertes do HCRP \\
\hline
\end{tabular}

\begin{tabular}{|c|c|c|}
\hline $\mathrm{ANO}$ & $\mathrm{N}^{\circ}$ & $\%$ \\
\hline 1997 & 6 & 40,0 \\
\hline 1998 & 4 & 26,7 \\
\hline 1999 & 3 & 20,0 \\
\hline 2000 & 2 & 13,3 \\
\hline TOTAL & 15 & 100,0 \\
\hline Forte: prontuári & & \\
\hline
\end{tabular}

Tabela VI - Distrbuição de pacientes do HCRP, segundo causa e ano da lesão medular e motivo de internação. no período entre 1997-2000 — Rظeirão Preto. 2001.

\begin{tabular}{|c|c|c|c|c|c|c|c|c|c|c|}
\hline \multirow[t]{2}{*}{ ANO CAUSA } & \multicolumn{2}{|c|}{1997} & \multicolumn{2}{|c|}{1998} & \multicolumn{2}{|c|}{1999} & \multicolumn{2}{|c|}{2000} & \multicolumn{2}{|c|}{ TOTAL } \\
\hline & $\mathrm{N}$ & $\%$ & $\mathrm{~N}$ & $\%$ & $\mathrm{~N}$ & $\%$ & $\mathrm{~N}$ & $\%$ & $\mathrm{~N}$ & $\%$ \\
\hline $\begin{array}{l}\text { Acriente autormobilistico } \\
\text { Ferimento por arra de fogo } \\
\text { Mergullo em águs rasas } \\
\text { Queda }\end{array}$ & $\begin{array}{l}1 \\
1 \\
1 \\
3\end{array}$ & $\begin{array}{r}6,6 \\
6,6 \\
6,6 \\
20,0\end{array}$ & $\begin{array}{l}1 \\
3 \\
- \\
-\end{array}$ & $\begin{array}{r}6,6 \\
20,0 \\
- \\
-\end{array}$ & $\begin{array}{l}- \\
- \\
-\end{array}$ & $\begin{array}{r}- \\
20,0 \\
- \\
-\end{array}$ & $\begin{array}{l}- \\
2 \\
- \\
-\end{array}$ & $\begin{array}{r}- \\
13,3 \\
- \\
-\end{array}$ & $\begin{array}{l}2 \\
9 \\
1 \\
3\end{array}$ & $\begin{array}{r}13,3 \\
60,0 \\
6,7 \\
20,0\end{array}$ \\
\hline TOTAL & 6 & 40,0 & 4 & 26,7 & 3 & 20,0 & 2 & 13,3 & 15 & 100,0 \\
\hline
\end{tabular}


Quadro 1 - Alterações fisio b́gicas registradas nos prontuários de pacientes com lesão medubar, no HCRP, e dias em que eles foram observadas após o trauma, mo período de 1997-2000 - Rbeirão Pre to, 2001.

\begin{tabular}{|c|c|c|}
\hline ALTERAÇÕES FISIOLÓGICAS & TOTAL DE PACIENTES & DIAS APÓS O TRAUMA \\
\hline 1- Arr eflexia de MMII & 2 & $5^{\circ}$ e $8^{\circ}$ dias \\
\hline Atrofia de MMII & 1 & $162^{\circ}$ dia \\
\hline 3- Bexiga N eurogêrica & 14 & 8 no $1^{\circ}, 4$ no $2^{\circ}, 1$ no $12^{\circ}$ e 1 no $55^{\circ}$ dia \\
\hline 4- Dimimux̧̧̃ão da F orça Muscular & 2 & $5^{\circ}$ e $8^{\circ}$ dia \\
\hline 5- Hipotoria de MMII & 1 & $1^{\circ} \mathrm{dia}$ \\
\hline 6- Hipotensão Postural & 4 & 2 no $3^{\circ}, 1$ no $21^{\circ}$ e 1 no $30^{\circ}$ dia \\
\hline 7- Ileo Par alifico (Obstipação) & 6 & 2 no $2^{\circ}, 1$ no $6^{\circ}$ e 1 m $20^{\circ}$ dia \\
\hline 8- Incontinêneia $\mathrm{F}$ ecal & 2 & $1^{\circ}$ dia \\
\hline 9- Infecção do Trato Urinário (ITU) & 3 & 1 no $3^{\circ}$ e 2 no $11^{\circ}$ dia \\
\hline 10- Pé eqüno & 2 & $13^{\circ}$ dia e 1 no $5^{\circ}$ mês após a lesão \\
\hline $11-$ Pico F ebril $\left(1^{\circ}\right.$ Pico $)$ & 9 & $4 \mathrm{~m} 2^{\circ}, 2$ no $3^{\circ}$, e 1 nos $6^{\circ}, 7^{\circ}$ e $32^{\circ}$ dias \\
\hline 12- Pneumoria & 2 & 1 no $3^{\circ}$ e 1 no $15^{\circ}$ dia \\
\hline 13- Priapismo & 1 & $1^{\circ}$ dia \\
\hline 14- Sudor ese/Palidez & 2 & $9^{\circ}$ e $10^{\circ}$ dias \\
\hline 15- Trombose Venosa Profunda & 2 & $56^{\circ} \mathrm{e} 86^{\circ}$ dias \\
\hline 16- Úlcera de Dectubito & 5 & 2 no $3^{\circ}, 2$ no $5^{\circ}$ e 1 no $4^{\circ}$ mês, após lesão \\
\hline 17-V ômito & 2 & $6^{\circ}$ e $9^{\circ}$ dias \\
\hline
\end{tabular}

\section{ANÁLISE DOS RESULTADOS E CONCLU- SÕES}

Através dos 15 prontuários pesquisados, notase que o perfil dos pacientes deste estudo, segundo idade e sexo, foi, na sua totalidade, do sexo masculino e de idade jovem, sendo que 11 pacientes $(83,4 \%)$ estão na faixa etária entre 16 e 30 anos e quatro $(26,4 \%)$ entre 31 e 45 anos. Desse total, $12(80,0 \%)$ são solteiros, dois $(13,4 \%)$ casados e $6,6 \%$ o que corresponde a um caso, não havia especificado o seu estado civil. Pode-se observar que, no período compreendido entre 1997-2000, o motivo das internações foram: nove $(60,0 \%)$ decorrentes de ferimento por arma de fogo, três $(20,0 \%)$ por queda, duas $(13,4 \%)$ por acidente automobilístico e uma $(6,7 \%)$ por mergulho em águas rasas. Seis $(40,0 \%)$ ocorreram no ano de 1997 , quatro $(26,7 \%)$ em 1998, três em $1999(20,0 \%)$ e duas $(13,4 \%)$ em 2000. Dos nove $(80,0 \%)$ causados por ferimento por arma de fogo, um (6.6\%) ocorreu em 1997, três $(20,0 \%)$ em 1998, três $(20,0 \%)$ em 1999 e dois $(13,4 \%)$ em 2000, o que demonstra um aumento significativo, em 1998 e 1999, e uma queda no ano de 2000. Quanto ao nível da lesão, duas $(13,3 \%)$ o) foram no nível da coluna cervical, $11(73,3 \%)$ no da coluna torácica, uma $(6,7 \%)$ no da coluna lombar e, em um $(6,7 \%)$ caso, não foi especificado; do número total de pacientes internados no HCRP, nove $(60,0 \%)$ foram encaminhados para a Unidade de Ortopedia, cinco $(33,3 \%)$ para a Clínica de Neurologia e um $(6,7 \%)$ para outra clínica, o que mostra que esses pacientes não possuem uma clínica específica para a sua hospitalização.

Comparando os dados obtidos com os verificados no informativo de trabalho da Spinal Cord lnjury Intormation Network, através de informações do Banco de Dados Nacional, ativo desde 1973, que capta dados de 24 estados norte-americanos, e citado por 
Furlan ${ }^{10}$, o trauma raquimedular também afeta, e principalmente os adultos jovens do sexo masculino. Para nós, autoras, o dado que difere do citado banco de dados, é o relativo ao nível neurológico da lesão que, nos estados norte-americanos é metade cervical (51 ,7\%) e metade lombar e sacral (46,7\%), e no HCRP, os níveis neurológicos encontrados foram $13,4 \%$ de lesões cervicais, $73,3 \%$ de torácicas e $6,7 \%$ de lesão lombar, o que pode estar relacionado com o tipo da violência vivenciada em Ribeirão Preto.

Ao observar o Quadro 1, o das alterações fisiológicas, verifica-se que as aqui ocorridas são semelhantes às citadas por diferentes autores ${ }^{(5,6,7)}$ e são mais comuns ocorrerem no lesado medular, por sua própria condição, que o torna mais suceptível no desenvolvimento de alterações graves e complicações imprevisíveis, embora, na maioria das vezes, haja a possibilidade de prevenção.

Desse modo, o aparecimento de certas alterações fisiológicas pode ser observado durante o período de internação e após a alta hospitalar, sendo que em três $(20,0 \%)$ casos resultou em nova hospitalização, um $(6,6 \%)$ por trombose venosa profunda e dois $(13,4 \%)$ por úlceras de pressão contaminadas.

Diante do quadro da lesão medular, como mostra a literatura, algumas alterações que pudemos constatar são esperadas para o paciente com tal lesão. Pudemos observar que a maioria deles apresentou bexiga neurogênica reffexa e não-reflexa (14 casos93,3\%), outros apresentaram arreflexia de membros inferiores (um caso-6,6\%), diminuição de força muscular (dois casos 3,4\%), hipotonia de membros inferiores (um caso-6,6\%), hipotensão postural (quatro casos-26,7\%), íleo paralítico (seis casos-40,0\%), in continência fecal (dois casos-13,4\%), infecção do trato urinário (três casos-20,0\%), pé eqüino (dois casos-13,4\%), pico febril (nove casos-60,0\%), pneumonia (dois casos-13,4\%), priapismo (um caso$6,6 \%$ ), sudorese/palidez (dois casos-13,4\%), trombose venosa profunda (dois casos-13,4\%), úlcera depressão (cinco casos-33,3\%) e vômito (dois casos-13,4\%), e a grande maioria dessas alterações ocorreu nos primeiros 15 dias após a entrada no hospital.

Quanto às necessidades básicas para o cuidado de enfermagem a esses pacientes, foi observado, nas Evoluções de Enfermagem, o cuidado com ações como prevenção a úlceras de decúbito, mudança de decúbito, banho no leito, cateterismo vesical intermitente ou de demora, curativos de úlcera de pressão e cicatrizes cirúrgicas, fleet enema, elevação de cabeceira. Em um prontuário, encontrou-se a anotação de uma enfermeira comunicando ao médico o quadro de anúria, e a conduta tomada.

Foi observada, também, a colocação de colchão d'água (um caso-6,6\%) e colchão caixa de ovo (dois casos-13,4\%), verificação de sinais vitais e, em todos os pacientes que apresentaram pico febril, a administração de medicamentos conforme prescrição médica, e, em apenas dois casos $(13,4 \%)$, fizeram-se compressas frias juntamente com a administração da terapêutica medicamentosa.

Dos 15 prontuários, somente em um $(6,6 \%)$ foram encontradas condutas e prescrições de enfermagem não relacionadas ao ato médico e de outros profissionais, como a vaporização contínua com água fervida, a verificação da diminuição de edema de membros infèriores e diminuição da distensão abdominal, embora, não se descrevesse a medida das circunferências e de quanto foi a diminuição. Também ocorreu a solicitação de exames, como tipo Rh e contra prova, pela enfermeira da referida instituição. Tais aspectos podem ser constatados e observados nas anotações abaixo:

"... hoje ocorreu discreta regressão do edema de MMSS e II, assim como da distensão abdominal. Mantemos cuidados respiratórios e antiescaras rigorosos, está em colchão caixa de ovos com vaporização com AF contínua". (Enfermeira/nome)

“... orientado ao funcionário o uso de cold cream no corpo; além da manutenção da vaporização contínua e cabeceira elevada". (Enfermeira/nome)

“...mantidos os MMII elevados (colocado suporte para pé equino bilateral) ". (Enfermeiral nome)

Através dessas anotações, pode-se observar a realização das ações de natureza terapêutica e propedêutica da enfermagem e as ações de natureza complementar de controle de risco, conforme classificação de Xavier et al.(1987) ${ }^{11}$. Apesar de terem sido encontradas em apenas um prontuário, e de ainda serem poucas e insuficientes para o quadro do paciente em questão, que se encontrava com TVP, febre, escaras, pneumonia e infecção do trato urinário hospitalar e hipotensão, já é um começo. Pode ser até que tenham ocorrido outras condutas, mas, elas nào foram registradas no prontuário do paciente. Frente ao encontrado, não dá para entender se as ações de en- 
fermagem aos outros pacientes com as alterações fisiológicas citadas anteriormente, no Quadro 1, foram realizadas e somente não foram anotadas, ou se nada foi feito.

Frente a isso, fica nosso questionamento: se nada foi feito, os profissionais sabem do que tratam as alterações apresentadas nesses pacientes, e como poderiam preveni-las ou amenizá-las? Não se pode prescrever cuidados para algo que não se conhece, se há conhecimento, porque não fazê-lo, complementando com a anotação do mesmo?

Outro aspecto importante e preocupante a ser relatado é o relativo à alta hospitalar e às orientações realizadas pelo profissional enfermeiro. Entendemos que o planejamento da alta hospitalar começa quando o paciente é admitido na instituição, pois seu preparo deve ser precoce para que se possa atender à necessidade de cuidados de que esse paciente necessitará no seu domicílio. O processo envolve a identificação das necessidades do paciente e o desenvolvimento de prescrições de cuidados para que sejam aprendidas e seguidas por ele e pelos cuidadores, evitando que surjam outras intercorrências e. assim que, novas internações ocorram. Seguem abaixo algumas anotações das altas hospitalares, encontradas nos prontuários médicos dos pacientes com lesão medular, embora em seis prontuários, não houvesse referência a tal lesão.

"Paciente preparado para sair de alta, vai de cadeira de rodas acompanhado pela mãe e namorada, que foram orientadas quanto as medicações, exames, auto-sondagem e cuidados com o material, em casa, de curativos, cuidados para a prevenção de escaras, exercícios respiratórios, uso de meia elástica e todos os retornos "(Enfermeira/nome).

"Orientado aos familiares quanto aos cuidados anti-escaras, quanto a SVA, alimentação e funcionamento intestinal." ( Enfermeira/nome).

"Paciente saindo de alta hospitalar acompanha do por familiares, foram orientados pela enfermeira." (Auxiliar de Enfermagem/nome).

"Alta hospitalar. Segue no ambulatório de ortopedia e bexiga neurogênica".

"Paciente saiu de alta, acompanhado pela madrasta, feito orientação quanto ao retorno no ambulatório de geriatria, exames e orientado quanto a troca da SVD no dia " (Enfermeira/nome).

"Paciente saindo de alta hospitalar, a mãe foi orientada quanto ao retorno". ( Enfermeira/nome).
"Orientado quanto a retorno e curativos que serào realizados em UBS." (Enfermeira/nome).

"Paciente saindo de alta, desce prontuários, adessograph e cartão. " (Enfermeira/nome).

Embora, em alguns prontuários apareça corretamente a relação dos cuidados a serem seguidos, preocupa-nos que a orientação apareça registrada apenas no momento da alta hospitalar e, com grande quantidade de informações. E sabido que quando o paciente recebe a notícia da alta hospitalar, por encontrar-se ansioso para o retorno ao seu domicílio, não consegue absorver totalmente as orientações passadas naquele momento. Por que não se trabalhar com esse processo, diariamente e gradualmente, durante hospitalização, com a vantagem de, assim, realizar todo um processo de aprendizagem com o paciente e o cuidador, mostrando-lhes diariamente, os cuidados que deverão tomar, quando estiverem em sua residência, e esclarecendo possíveis dúvidas que possam surgir?

Recomendamos, então, que o preparo para a alta hospitalar seja o mais precoce possível e envolva o binômio paciente/família.

\section{CONSIDERAÇÕES FINAIS}

A realização deste estudo possibilitou-nos uma visão mais ampla cio contexto que envolve o cuidado ao paciente com lesão medular, internado na fase aguda da lesão.

Acredita-se ser importante, em enfermagem, um programa de educação continuada, de modo a garantir para toda a equipe a atualização do conhecimento das necessidades de cada paciente e de sua família e da etapa pela qual se está passando. Além disso, levar todos os integrantes da equipe de enfermagem à conscientização sobre a importáncia da anotação em prontuário, que quando bem elaborada, apresenta elementos valiosos para o diagnóstico das necessidades do paciente e para o planejamento e avaliação da assistência prestada.

Além disso, este trabalho indica a necessidade da elaboração de um roteiro específico pai direcionar o cuidado que deve ser dado ao paciente com lesào medular, roteiro esse que deve permanecer na unidade para ser consultado toda vez que dúvidas ocorram ou para subsidiar a Sistematização da Assistência de Enfermagem (SAE). 
SARTORI NR \& MELO MRAC. Needs regarding the hospital care provided to spinal cord injured patients. Medicina, Ribeirão Preto, 35: 151-159, apr./june, 2002

ABSTRACT: Study type: descriptive and documentary work. Purpose: to identify the incidence of complications and the care provided by the nursing team to spinal cord injured patients. Method: the records of spinal cord injured patients were analyzed according to the ICD 10, from 1997 to 2000. Data were obtained through the Hospital File Service, through the request of a morbidity report. Afterwards, authors elaborated an instrument containing patient's identification, basic data on clinical history and physiological alterations, as well as the day these alterations occurred and the care provided by the nursing team. Data were grouped and analyzed through a table and a chart. Results: authõrs observed that these patients were really susceptible to complications as infections, neurogenic bladder, paralytic ileus, priapism, profound venous trombosis, among others. They also found, through the annotatioris of the nursing team, the inexistence of specific care to these complications besides the basic care and the actions of propaedeutic and therapeutic nature that are complementary to the medical act. Authors also verified that six records did not present annotations about discharge orientations and that the notes in nine records were not enough regarding the care that these patients and the ones who provide their care would need at home in order to prevent these complications. Considerations: this study facilitated a broader view of the context involving the spinal cord injured patient. Authors believe that it is important to develop a nursing continuing education program in order to assure that the members of the team will update their knowledge about the needs of each patient and the stage they are going through.

Uniterms: Somal Cord Injury. Nursing Care. Orthopaedic Nursing.

\section{REFERÊNCIAS BIBLIOGRÁFICAS}

1 - MELO MRAC FERRAZ CA, SOUZA CR \& LAVRADOR MAS. Estudo do quadro de morbidade da unidade de internação de ortopedia. Medicina, Ribeirão Preto, 33: 73-81, 2000.

2- PITZEN P \& RÕSSLER H. Manual de ortopedia. 13 ed. Tradução de Clemente de Almeida Moura, Horst H. Werver. Atheneu, São Paulo, 1981.

3 - FARO ACM. Estudo das alterações da função sexual em homens paraplégicos. Dissertação de Mestrado, Escola de Enfermagem da USP, São Paulo, p 1-98, 1991.

4 - HOPPENFELD 5 Neurologia para ortopedistas. Tradução de Ubirassi Silveiro Siqueira e Fernando Pompeu. Cultura Médi ca, Rio de Janeiro, 1985.

5- SARAIVA RA; PIVA JUNIOR L; PAZ JUNIOR AC \& PACHECO MAR. As bases fisiopatológicas para a anestesia no pacien te com lesão medular. Rev Bras Anestesiol,45, 387-398, 1995.

6 - SMELTZER SC \& BARE BG. Tratado de enfermagem mé dicocirúrgica. 8 ed. Guanabara Koogan, Rio de Janeiro, 1998.

7- OSBORN JR; ROGERS HH \& POUSADA L. Enfermagem de emergência - um manual prático. Tradução de Ana Ma ria Müller Magalhães, Artes Médicas, Porto Alegre, 1992. J
8- FACHIN O. Fundamentos de metodologia. 3 ed. Sarai va. São Paulo, 2001.

9-ORGANIZAÇÃO MUNDIAL DA SAÚDE. ORGANIZAÇÃO PANAMERICANADE SAÚDE. MINISTÉRIO DASAÚDE. Ma nual de Classificação Internacional de Doenças, Le sões e Causas de Obito. 10 Revisão. São Paulo, Uni versidade de São Paulo, 1998.

10- FURLAN MLS. Indentificação das práticas de autocuidado referentes ao funcionamento intestinal em pacientes com trauma raquimedular. Dissertação de Mestrado, Escola de Enfermagem de Ribeirão Preto da USP, Ribeirão Preto, p 1127, 2001.

11 - XAVIER IM; SOUZAAMA; ARAUJO EC; RODRIGUEZ NETO E; SOUZA EO; BRICENO G; SANTOS 1; AZEVEDO J; COMINO LBS; CHRISTÓFARO MAC; PUNTEL MC; ROSSI MJ; FRIEDLANDER MR; SILVANF; CHOMPRÉ RR; GIRARDI SN; BARROS 5 \& VIEIRA TCV Subsídios para a conceituação da assistência em enfermagem rumo à Reforma Sanitária. Rev Bras Enferm, Brasília, 40: 177-180, 1987.

Recebido para publicação em 27/12/2001

Aprovado para publicação em 28/06/2002 


\section{ANEXO 1}

\section{1- DADOS PESSOAIS}

$\mathrm{N}^{\circ}$ Registro (HCRP):

Sexo:

Idade:

Estado Civil:

Unidade de Internação:

Data da Internação:

Data da Alta:

Diagnostico médico:

2- DADOS DA HISTÓRIA CLÍNICA (Nível e tempo da lesão e motivoda lesão)

3- ALTERAÇÕES REGISTRADAS NO PRONTUÁRIO MÉDICO

\begin{tabular}{|l|l|l|l|}
\hline Dias após internação & Intercorrências & $\begin{array}{c}\text { Atividade de } \\
\text { enfermagem realizada }\end{array}$ & Categoria profissional \\
\hline & & & \\
\hline & & & \\
\hline & & & \\
\hline & & & \\
\hline
\end{tabular}

